\title{
Analyzing a putative enhancer of optic disc morphology
}

\author{
Vladimir Babenko ${ }^{1,2^{*}}$ (D), Roman Babenko ${ }^{1,2}$ and Yuri Orlov ${ }^{1,2,3}$ \\ From 11th International Young Scientists School "Systems Biology and Bioinformatics" - SBB-2019 \\ Novosibirsk, Russia. 24-28 June 2019
}

\begin{abstract}
Background: Genome-wide association studies have identified the CDC7-TGFBR3 intergenic region on chromosome 1 to be strongly associated with optic disc area size. The mechanism of its function remained unclear until new data on eQTL markers emerged from the Genotype-Tissue Expression project. The target region was found to contain a strong silencer of the distal $(800 \mathrm{~kb}$ ) Transcription Factor (TF) gene GFl1 (Growth Factor Independent Transcription Repressor 1) specifically in neuroendocrine cells (pituitary gland). GFI1 has also been reported to be involved in the development of sensory neurons and hematopoiesis. Therefore, GFII, being a developmental gene, is likely to affect optic disc area size by altering the expression of the associated genes via long-range interactions.
\end{abstract}

Results: Distribution of haplotypes in the putative enhancer region has been assessed using the data on four continental supergroups generated by the 1000 Genomes Project. The East Asian (EAS) populations were shown to manifest a highly homogenous unimodal haplotype distribution pattern within the region with the major haplotype occurring with the frequency of 0.9. Another European specific haplotype was observed with the frequency of 0.21. The major haplotype appears to be involved in silencing GFl/repressor gene expression, which might be the cause of increased optic disc area characteristic of the EAS populations.

The enhancer/eQTL region overlaps AluJo element, which implies that this particular regulatory element is primatespecific and confined to few tissues.

Conclusion: Population specific distribution of GFI1 enhancer alleles may predispose certain ethnic groups to glaucoma.

Keywords: GWAS, Enhancers, Optic disc size, CDC7, TGFB3, Glaucoma, 1000GP, GFI1, Alu, GTEx

\section{Background}

Genome-wide association studies (GWAS) identified thousands of common single nucleotide polymorphisms (SNPs) associated with complex diseases and quantitative traits [1]. These SNPs affect a trait in different ways. They cause an amino acid substitution, change the

\footnotetext{
* Correspondence: bob@bionet.nsc.ru

${ }^{1}$ Institute of Cytology and Genetics, Lavrentyeva 10, Novosibirsk 630090, Russia

${ }^{2}$ Novosibirsk State University, Pirogova Str 2, Novosibirsk 630090, Russia Full list of author information is available at the end of the article
}

splicing process, and change the transcription rate or translational efficiency [2]. Identified variants were located in various regions of the genome including coding and regulatory genes regions. A large part of SNPs significantly associated with complex traits are located in non-coding regions: about 45 and $43 \%$ of such SNPs are located in introns or intergenic regions, respectively [1].

Gene regulation studies provide convincing evidence that a significant part of non-coding GWAS loci function as enhancers that can regulate specific genes up to $1 \mathrm{Mb}$ away. The enhancers exhibit Transcription Factor

(c) The Author(s). 2020 Open Access This article is licensed under a Creative Commons Attribution 4.0 International License, which permits use, sharing, adaptation, distribution and reproduction in any medium or format, as long as you give appropriate credit to the original author(s) and the source, provide a link to the Creative Commons licence, and indicate if changes were made. The images or other third party material in this article are included in the article's Creative Commons licence, unless indicated otherwise in a credit line to the material. If material is not included in the article's Creative Commons licence and your intended use is not permitted by statutory regulation or exceeds the permitted use, you will need to obtain permission directly from the copyright holder. To view a copy of this licence, visit http://creativecommons.org/licenses/by/4.0/ The Creative Commons Public Domain Dedication waiver (http://creativecommons.org/publicdomain/zero/1.0/) applies to the data made available in this article, unless otherwise stated in a credit line to the data. 
Binding Sites (TFBS) clusters, open chromatin and corresponding histone marks. Thus, TFBS genome-wide locations identified by ChIP-Seq data, cell line specific chromatin state landscape, DNAse hypersensitive sites (DHS) at the intronic/intergenic sites can point to the enhancer loci. The results on the phenomenon were outlined in papers connected with the emergence of chromatin state segmentation routine [3-5]. As a practical outcome therein, the authors were able to associate a range of either target or tightly linked noncoding SNPs from GWAS studies with chromatin states corresponding to strong enhancers [4]. In particular, they identified two SNPs strengthening tissue specific transcription factor binding sites: SNP rs9374080 associated with red blood cells (RBC) phenotype in close proximity (100 bp) to a strong enhancer in the K562 cell line, which reinforces the binding motif for $G F I 1 B$, a predicted repressor in K562, by strengthening it. Another lupus-associated SNP (rs9271055) locates within a lymphoblastoid (GM12878) strong enhancer and strengthens the binding motif for ETS1, a predicted activator of lymphoblastoid enhancers [4].

With an advent of Hi-C technology [6], Assay for Transposase Accessible Chromatin (ATAC-Seq) [7], and large-scale Multiplex Reporter Assays (MRA) [8], the enhancers identification was given renewed impetus resulting in the accumulation of a plethora of enhancer loci. VISTA, an experimentally verified enhancer source (https://enhancer.lbl.gov/) [9] includes around 2000 entries. Modern enhancer databases based on circumstantial evidence include entries on several million enhancers [10]. GeneHancer database appears to be the most comprehensive enhancer resource to-date [11]. It uses more than 1 million enhancers compiled from seven different genome-wide databases: the Encyclopedia of DNA Elements (ENCODE), Z-Lab Enhancer-like regions (http://zlab-annotations.umassmed.edu/enhancers/), the Functional Annotation of the Mammalian Genome (FANTOM) project [12], the Ensembl regulatory build [13], dbSUPER super-enhancers [14], EPDnew promoters [15] and UCNEbase of ultraconserved non-coding elements [16].

The filtering criteria applied at the GeneHancer pipeline (as of 2018 version) underscores 285,000 enhancer loci, with 94,000 having more than one source of evidence ("double elite").

The next crucial step in enhancer annotation (even more important than identification of enhancer itself) is annotating the genes associated with a particular enhancer. The Gene-GeneHancer associations were ascertained using 5 criteria/evidence sources:

1) eQTLs (expression quantitative trait loci) from The Genotype-Tissue Expression Consortium (GTEx; https://www.gtexportal.org/home/; [17]; version v6p)

2) Capture Hi-C promoter-enhancer long range interactions
3) FANTOM5 eRNA-gene expression correlations

4) Cross-tissue expression correlations between a transcription factor interacting with a GeneHancer and a candidate target gene;

5) Distance-based associations, including several approaches:

a. Nearest neighbors, where each GeneHancer is associated with its two proximal genes

b. Overlaps with the gene territory (intragenic)

c. Proximity to the gene TSS $(<2 \mathrm{~kb})$

In particular, eQTL database is a large-scale project of GTEx consortium [17]. It comprises 1,5 mln eQTL SNPs across 44 tissues (v.6p). Notably, GWAS SNPs often overlap the eQTL ones while not being themselves causative SNPs [18]. Thus, GWAS, chromatin state [19], and eQTL data complement each other with a task of elucidating causative SNPs in gene-enhancer interaction.

Speaking of gene-enhancer associations, there are currently numerous examples undermining the view, popular several years ago, that the majority of GWAS signals detected in intergenic/intronic regions are due to the functions of the nearest genes. For example, FTO intronic locus linked to obesity phenotype was proved to be a $(800$ $\mathrm{kb})$ distal IRX3 gene enhancer [20]. A range of intergenic enhancers is annotated in VISTA database [9].

This study is focused on optic disc area as a glaucoma-related trait. Previous research efforts have established 3 major traits impacting glaucoma risk rate: optic disc size (area), optic disc morphology, and retinal nerve fiber layer (RNFL) thickness [21].

At least four genome-wide association studies known to date (GWAS) [21-24] have demonstrated with high significance that rs1192415is associated with optic disc parameters ( $P<8 \mathrm{E}-17$; 3E-28; 8E-56; 6E-81, respectively).

In particular, SNPs rs1192415, rs4658101, and rs1192419 significantly associated with optic disc area have been localized between genes CDC7 and TGFBR3 [21-25]. The former encodes a cell division cycle protein with kinase activity that is critical for the G1/S transition and the latter encodes a transforming growth factor. The previous association of the above GWAS markers was majorly to the TGFBR3 gene [25]. This study will focus on the inferred function of the locus as GFI1 gene enhancer.

The ultimate aim of the project was to analyze population specific distribution of genome-wide significant SNPs for optical disc area, which was not approached before.

\section{Results}

Choosing the region of interest

Several GWAS optic disc area projects identified ATOH7 gene and CDC7-TGFBR3 intergenic region as 
major determinants of optic disc area. According to the most recent meta-analysis studies the rate of associations is $P<1 \mathrm{E}-112$ and $P<6 \mathrm{E}-81$ for ATOH7 and CDC7TGFBR3 intergenic region, respectively [24]. While ATOH7 gene was shown to be part of the embryonic optic disc area gene regulatory network, the locus association and mechanism of gene causality remained unclear. According to GWAS data, the locus represents a short $4 \mathrm{~kb}$ region starting with rs1192415 and ending with rs1192419 (Fig. 1).

The GTEx eQTL data repository emerged in 2015 [17] and proved to be the largest eQTL resource to date. It comprises $1.6 \mathrm{mln}$ unique eQTL associations across 43 tissue types [17]. We queried GTEx data across our $4 \mathrm{~kb}$ region of interest (chr1 $92.077 \mathrm{mb}-92.082 \mathrm{mb})$. It was found that the single tissue (pituitary gland) exhibits a set of SNPs associated with GFIIgene (eQTL $p$-value: $1.7 \times$ $10^{-5}$ ) located $840 \mathrm{~kb}$ downstream the target locus. GFI1 gene is a growth factor gene largely involved in early embryogenesis of a subset of tissues, including retinal.

\section{GeneHancer elements associated with GFI1}

From GeneHancer database nine enhancer elements manifesting GFI1 as a target and located in the vicinity of GFI1 (all GeneHancer elements are cis relative to a target gene(s)), GH01J091608 annotation corresponds to the target enhancer element. Single evidence of association of the latter with GFI1 was ascertained via GTEx eQTL data as the only source.

Still, it maintains active state in 19 of 68 ENCODE cell lines/tissues (see ENSR00000009735 at www.ensembl.org), mostly related to embryonic stage (A549, Fetal Adrenal Gland, Fetal Stomach, H1-mesenchymal, H1-trophoblast, HMEC, HSMM, HSMMtube, HeLa-S3, IMR90, Left Ventricle, Lung, MSC (VB), NH-A, NHDF-AD, NHEK, NHLF, Osteoblasts, Placenta). The correspondent eQTL SNPs from GTEx exhibit a repressive effect for GFI1 in only one of 43 tissues considered (pituitary gland). GeneHancer and Gene share a Topological Associated Domain (TAD) with evidence in $3 / 20$ biosamples ( $\mathrm{HiC}$ data).

\section{Distribution of alleles elucidated as eQTL locus}

On the basis of SNPs data from both GWAS and GTEx we compiled a set of 15 SNPs (Table 1) evenly spanning within $4 \mathrm{~kb}$ (Fig. 1).

The profiles of MAF alleles across all supergroups (Fig. 2) underscore that GWAS related haplotype outstands from haploblock linkage in all supergroups other than EAS. Overall, the region maintains a high linkage rate $(r>0.9)$ for all eQTL SNPs, as well as within GWAS $\operatorname{SNPs}(r>0.9)$.

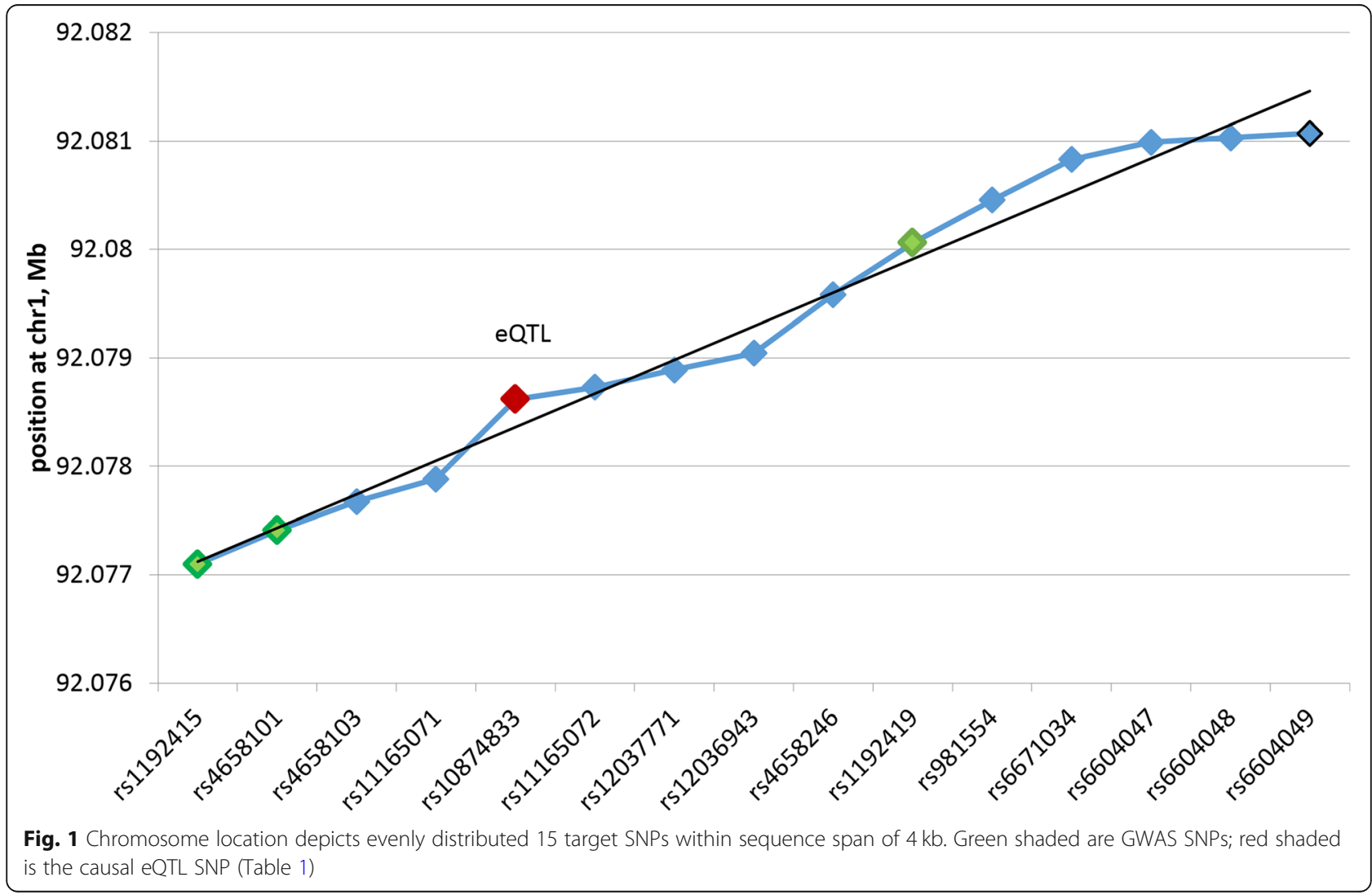


Table 115 SNPs minor frequencies for merged GWAS and GTEx SNP sets considered in the study for European (EUR), South Asian (SAS), East Asian (EAS) and African (AFR) supergroups

\begin{tabular}{llllll}
\hline & EUR & SAS & EAS & AFR & maf allele \\
\hline rs1192415** & 0.173 & 0.3037 & 0.1399 & 0.2103 & $\mathrm{G}$ \\
rs4658101*** & 0.175 & 0.3037 & 0.1399 & 0.3829 & $\mathrm{~A}$ \\
rs4658103 & 0.3867 & 0.3507 & 0.1399 & 0.4276 & $\mathrm{G}$ \\
rs11165071 & 0.3867 & 0.3517 & 0.1399 & 0.4276 & $\mathrm{G}$ \\
rs10874833 & 0.3877 & 0.3517 & 0.1399 & 0.4375 & $\mathrm{C}$ \\
rs11165072 & 0.3877 & 0.3517 & 0.1399 & 0.4375 & $\mathrm{~T}$ \\
rs12037771 & 0.3946 & 0.3569 & 0.1399 & 0.4375 & $\mathrm{~T}$ \\
rs12036943 & 0.3877 & 0.3517 & 0.1399 & 0.4375 & $\mathrm{~A}$ \\
rs4658246 & 0.3877 & 0.3517 & 0.1399 & 0.4375 & $\mathrm{~T}$ \\
rs1192419** & 0.1759 & 0.3047 & 0.1339 & 0.2927 & $\mathrm{~A}$ \\
rs981554 & 0.3857 & 0.3282 & 0.1359 & 0.4355 & $\mathrm{~A}$ \\
rs6671034 & 0.3867 & 0.3272 & 0.1339 & 0.4365 & $\mathrm{~A}$ \\
rs6604047 & 0.3867 & 0.3262 & 0.1339 & 0.4365 & $\mathrm{~A}$ \\
rs6604048 & 0.3867 & 0.3272 & 0.1339 & 0.4365 & $\mathrm{~T}$ \\
rs6604049 & 0.3867 & 0.3272 & 0.1339 & 0.4365 & $\mathrm{~A}$ \\
\hline **GAs SNPs with minimal $P$ values: $(P<8$ E-17;6E-81; 8E-56), respectively
\end{tabular}

**GWAs SNPs with minimal $P$ values: $(P<8 \mathrm{E}-17 ; 6 \mathrm{E}-81 ; 8 \mathrm{E}-56)$, respectively ${ }^{a}$ GTEx causal SNP

The highly interlinked $4 \mathrm{~kb}$ locus has proved to be eQTL allele (Fig. 2; blue shaded) for the GFI1 gene. Three GWAS SNPs differed in their MAF values from the core haplotype in EUR populations (Fig. 2; enlarged blue markers, green shadowed), and thus are non- equally linked with others $(r<0.8)$ in the supergroups other than EAS (full linkage overall), while highly interlinked with each other $(r>0.9)$ in European and South Asian supergroups. SNPs are sorted according to their order on the chromosome.

\section{Distribution of haplotypes}

We retrieved 15-fold haplotypes frequencies from 1000G phased data for each of the supergroups (Table 2) that are higher than 0.1 total across all populations. Due to a high linkage rate within the locus, three haplotypes encompass $>95 \%$ of variance in all supergroups except AFR (Table 2). All eQTL SNPs are completely linked and represent 2 dichotomous alleles. The major haplotype exhibits no variation and is interlinked with GWAS SNPs, so all variations reside in minor allele spectra. The haplotypes composition manifests overlapping GWAS 3-fold haplotype with completely linked 12-fold eQTL haplotype. Thus, as a major (tag) haplotype we may set the highest associated with trait GWAS polymorphism rs4658101 (Table 1) complemented with most 'enhancer' manifested, presumably casual, eQTL SNP rs10874833.

After performing PCA for Table 2 data, we found the haplotype spectra quite distinctly distributed across 4 major continental supergroups (Fig. 3), implying it can affect specifically the Asian populations due to a single mode haplotype distribution with highly linked optical disc size allele and eQTL SNPs. Also, we may underscore the unique European-descent haplotype, which apparently disrupts the silencer site (eQTL) (Fig. 3).

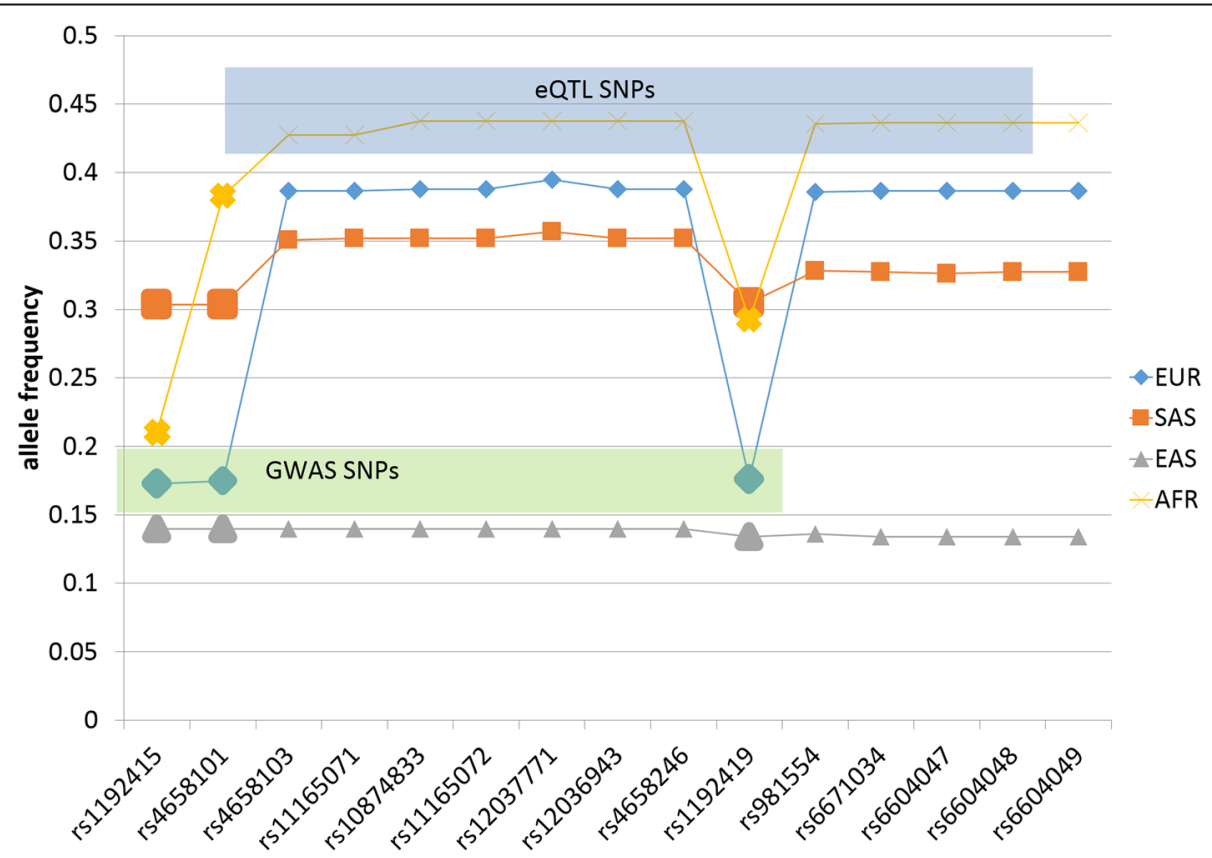

Fig. 2 Graphic representation of MAF frequencies from Table 1 for European (EUR), South Asian (SAS), East Asian (EAS) and African (AFR) supergroups. GWAS SNPs are plotted with enlarged markers 
Table 2 Distribution of top haplotypes frequencies in 4 major supergroups $^{a}$

\begin{tabular}{llllll}
\hline Haplotype (15 letters) & 'Tag' haplotype & AFR & EAS & EUR & SAS \\
\hline AGAAGGCGAGGGGAG & GG & 0.562 & 0.86 & 0.605 & 0.642 \\
GAGGCTTATAAAATA & AC & 0.209 & 0.132 & 0.172 & 0.28 \\
AGGGCTTATGAAATA & GC & 0.043 & 0 & 0.21 & 0.045 \\
SUM: & & 0.814 & 0.992 & 0.987 & 0.967
\end{tabular}

${ }^{a}$ Supergroup denotations are given in Fig. 2. GWAS SNPs are marked with bold, eQTL causal SNP is marked in bold Cyrillic. The first allele is a major one ( $f>0.5$ in all supergroups), two others are minor ones. The 'tag' haplotype represents convoluted GWAS/eQTL 2-letter haplotype of tag SNPs (pos. 5:rs4658101, pos. 10:rs10874833). Two African-specific low frequency haplotypes AAGGCTTATGAAATA (0.099) and AAGGCTTATAAAATA (0.07) were omitted from consideration according to selection criterion

\section{Allele dosage estimation}

If the causal eQTL SNP rs1087483 G is considered as the risk one (it doesn't decrease GFI1 expression) and co-dominant, then the estimated homozygous state in EAS would be p $(\mathrm{GG})=0.74$ within the major haplotype. In contrast, in EUR supergroup it would be p (GG) = 0.36, 2 times lower than in EAS. Overall, the major allele in EUR, AFR, SAS supergroups occurs with approximately similar frequency (Table 2).

\section{Discussion}

The advance of the resources and technologies on enhancer validation made it possible to move the task of annotating GWAS associated loci toward a new stage. While the information on the enhancers, and especially, their target regions is still far from complete, the range of targets conceived to date have been ascertained.

We studied the CDC7-TGFB3 intergenic region to elucidate the exact target of a $4 \mathrm{~kb}$ loci enriched with GWAS SNPs associated with optical disc size, at the same time manifesting eQTL SNPs pointing to GFI1 gene target at least in pituitary gland tissue based on eQTL GTEX database evidence. We justify the likelihood of this enhancer to be also active in embryonic retinal tissues by presenting two observations.

First, we assessed the tissue specific distribution of eQTL regions against the number of tissues they are active. As we can see from Fig. 4, there is a strong tenfold overrepresentation of single-tissue eQTL regions not fitting exponential distribution, otherwise highly concordant $\left(R^{2}=0.93, d f=42 ; p<2.6 \mathrm{E}-51\right)$.

We assume that overabundance of single - tissue enhancers implies they may get activated at certain embryonic stages, or in some specific tissues not currently presented in GTEX tissue panel.

While there is a promising approach for detecting embryonic enhancers reported quite recently [26], it may require some time to address the issue explicitly.

As the second observation corroborating the hypothesis stated above, we correspond that none of the

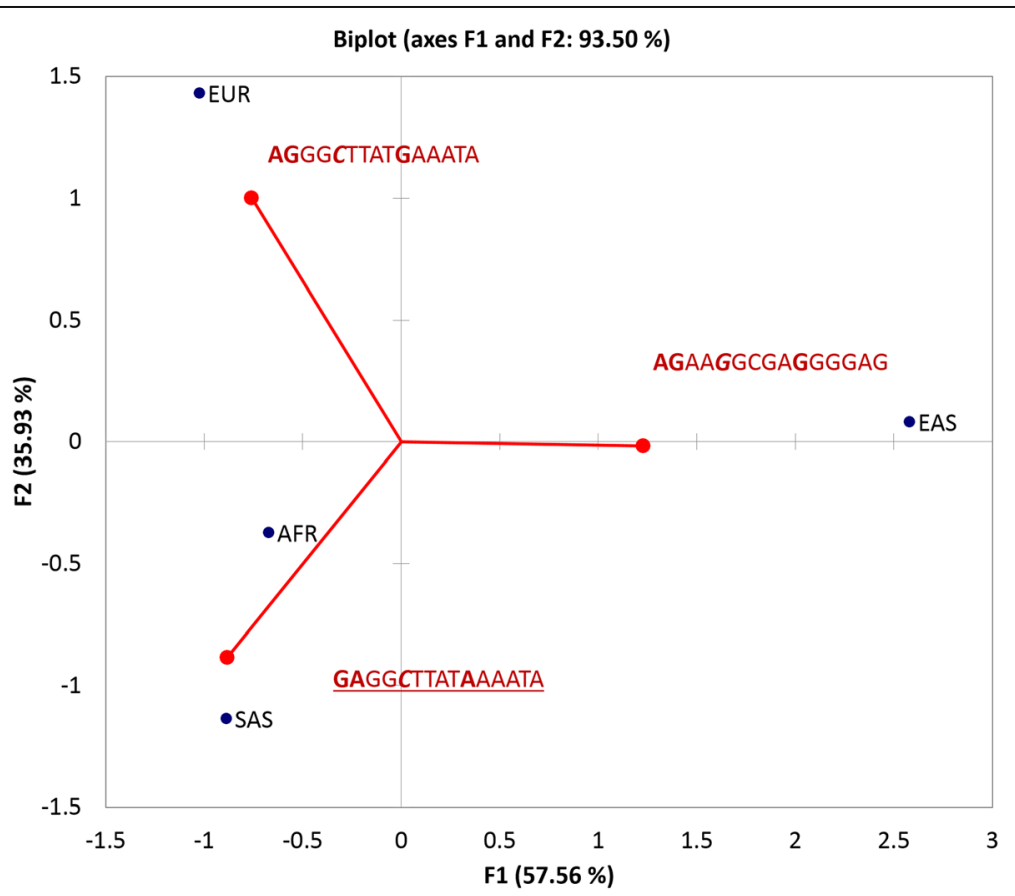

Fig. 3 PCA plot of 3 major haplotypes (Table 2) in European (EUR), South Asian (SAS), East Asian (EAS) and African (AFR) supergroups. GWAS SNPs (Table 1) are marked in bold. eQTL casual repressive SNP rs1087483 G- >C (pos. 5) is marked in bold italics. Underlined is the high-risk allele according to GWAS reports. The low-risk allele (GWAS haplotype: AGG; on the right) is highly elevated at EAS supergroup, and is present at least at $50 \%$ in the others (Table 2) 


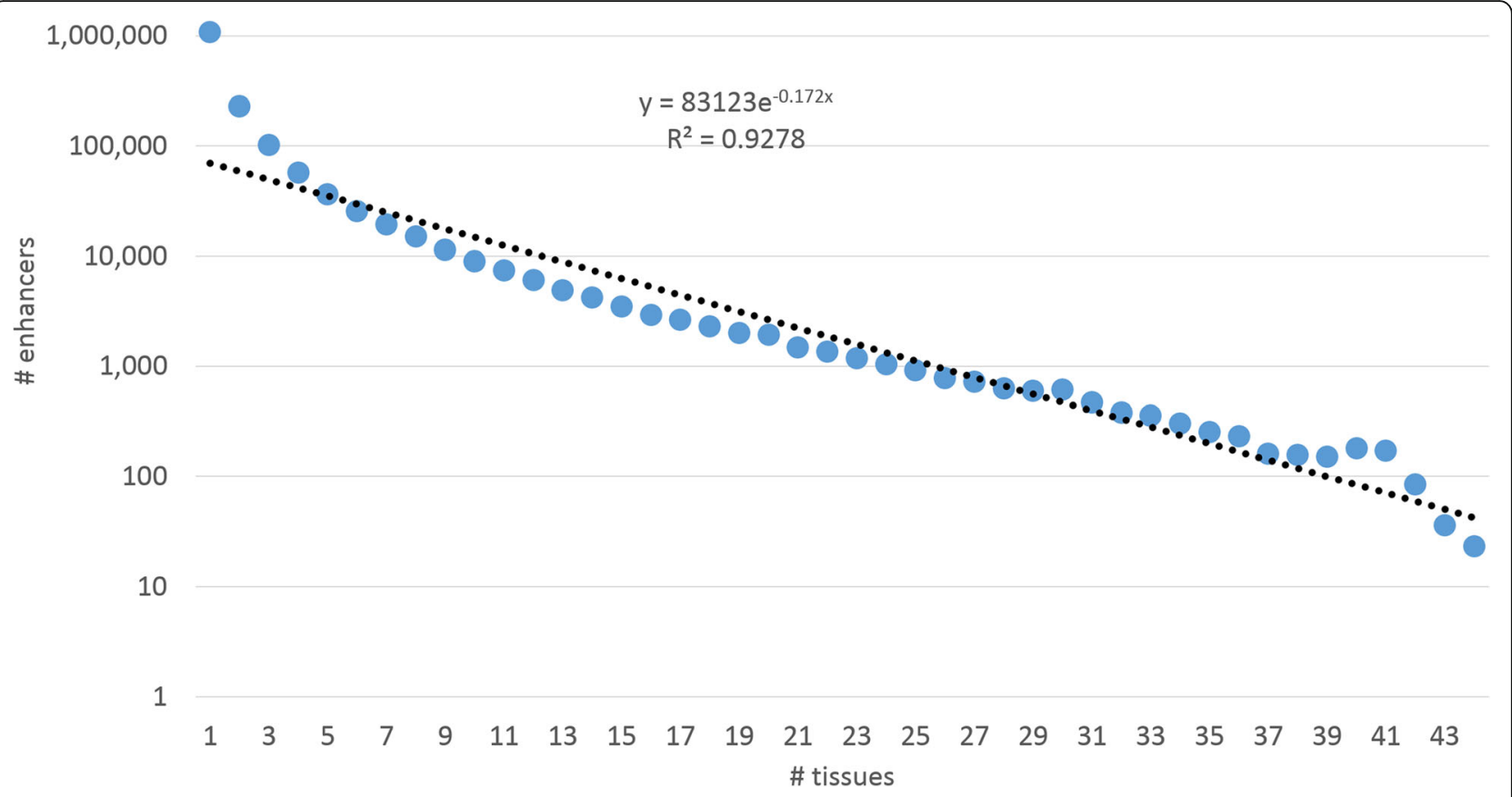

Fig. 4 Distribution of enhancers' number given the number of tissues it is active in, and its exponential approximation based on GeneHancer elements database

distinct enhancers reported in GeneHancer database overlap each other. That means that virtually no random overlapping may occur for the embryonic-related CDC7TGDBR3 enhancer and pituitary specific one.

Based on these observations, we speculate that the pituitary specific enhancer may possibly get activated in embryonic nerve tissue in the course of optic disc development.

GFI1 was first ascertained as an oncogene in lymphomas [27, 28]. Further studies showed that GFI1 mRNA is expressed in many precursors that give rise to neuronal cells during embryonic development in mouse [29]. GFI1 mRNA is expressed both in the CNS and PNS, featuring many sensory epithelia cells including the developing retina, the eye, presumptive Merkel cells, the lung and hair cells of the inner ear [30]. It has homologues in fly (senseless) and worm (pag-3).

GFI1 was proved to maintain tissue specific distal enhancers up to $100 \mathrm{~kb}$ in early hematopoietic cells [31], later ascertained to be involved in a complex interplay of enhancers and silencers, as well as genes within Gene Regulation Networks in hematopoietic lineage specification [32]. Similarly, GFI1 plays a crucial role during embryogenesis of other tissues [33]. During embryogenesis the enhancers' network is one of the expanded regulatory networks and often incorporates Transposable Elements (TE) including Alus, which later become repressed [34-36]. Currently, GeneHancer resource provides evidence (eQTL only) for the target region as an enhancer of GFI1 [11].
The tissue restricted enhancer in the region spanning target GWAS SNPs (Fig. 5; yellow track for hESC cell line) comprises several TFBS, including CTCF and $p 300$, which regularly accompany $\mathrm{Hi}-\mathrm{C}$ looping factors [11]. It looks probable that the enhancer site changes chromatin context in the locus vicinity upon activation and accomplishes interaction of the locus with the promoter region of GFI1 via Hi-C mechanism. Since GWAS SNPs are also heavily interlinked with eQTL SNPs which decrease the expression of GFI1 (Table 1), we speculate that three GFI1 expression alteration hypotheses are possible: 1) GWAS SNPs are the cause of abrogation of the aforementioned looping, e.g. by disruption of CTCF or other binding sites, resulting in the alteration of GFI1 expression; 2) GFI1 binding site located in AluJo/AluSo segment [37] is probably involved in local autoregulation of GFI1 expression. Note that the causative eQTL SNP rs10874833 is only 10 bp away from the core consensus of GFI1 within AluJo ('AATC'; [37]) and may affect the binding affinity of GFI1 to this site; 3) AluJo comprises CTCF binding site which is altered by eQTL SNP followed by long-range interaction abrogation (loop disruption).

Since zinc finger protein GFI1 contains at least 3 zinc finger motifs, the consensus should be much more extended, and it is partially reflected in overall consensus length of 10 positions in Hocomoco database (Additional File 1), though other positions are not informative probably due to (single dependence) weight matrix 


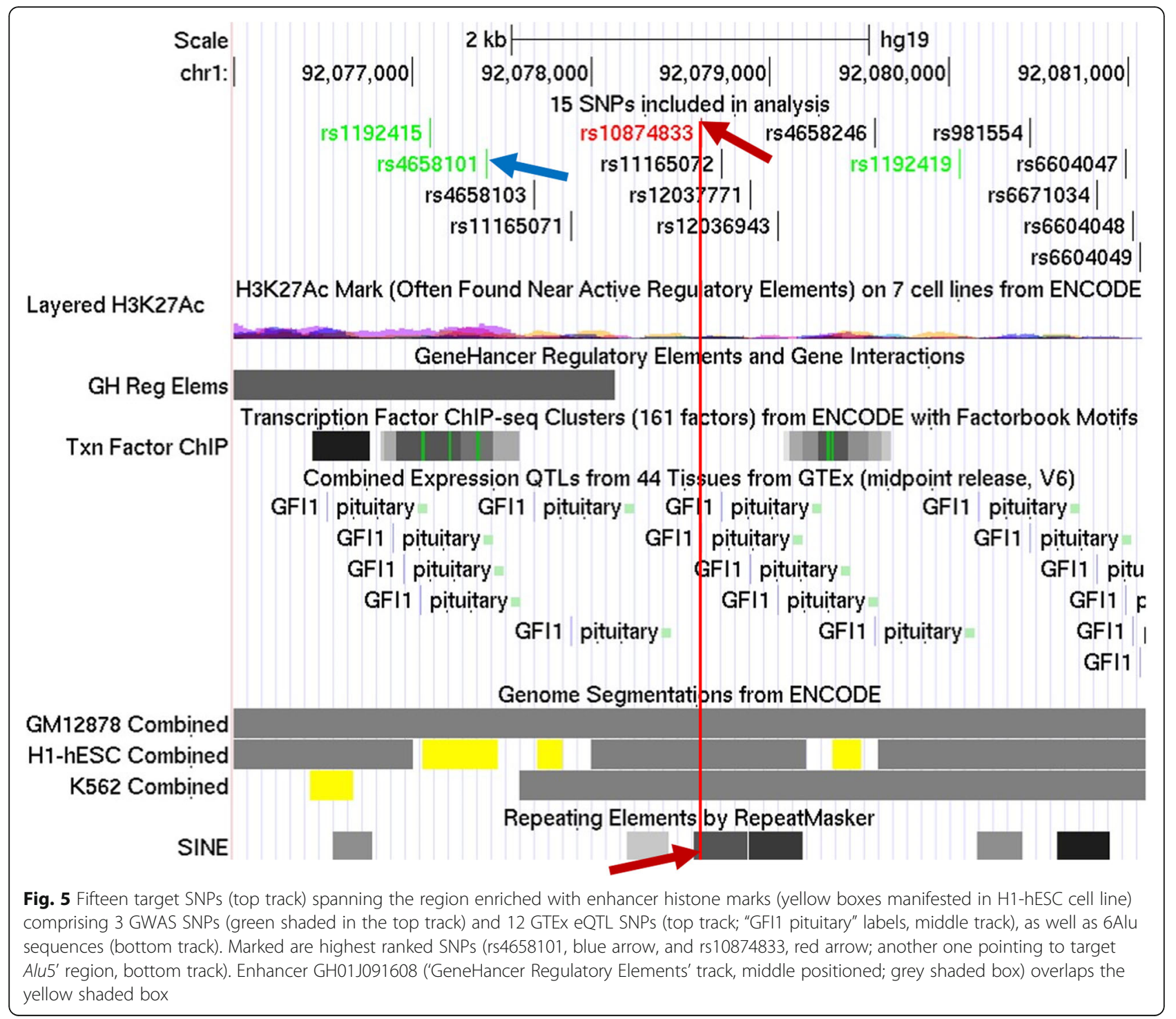

approach utilized in Hocomoco [38], not accounting for possible interaction of the positions, including palindromic structure preference [39], and, more importantly for the ZNF-related TFs, the orientation-related specifics mediated by specific pairwise position correlations [40]. It should be noted the Alu related abundance of GFI1 motif.

The target locus maintains specific structure comprising tandem Alu pair flanked with enhancer- specific H3K27ac histone marks (Fig. 5). At least 4 eQTL SNPs fall into 3 Alus, including the causal eQTL SNP (rs1087483; Fig. 5, red arrow). Since eQTL SNPs are highly interlinked in the region in all supergroups, we may speculate that since the highest ranked SNPs, both GWAS and eQTL ones (rs4658101 and rs10874833, respectively; Fig. 5) belong to the region flanked with enhancer border marks (Fig. 5, yellow boxes of chromatin segmentation track), other SNPs outside the region are presumably non-causative.

As mentioned above, we should add that GFI1 TFBS residing in a transposable element like $A l u$ is an annotated event $[29,38]$, and suggests multiple factors, including DNA methylation and heterochromatization that may play a role in accessibility of such a site in a tissue and stage-specific manner. It is worth noting that Alu TEs proved to be involved in developmental stages [37], and comprise the following TFBSs: GFI1 (V\$GFI1.01), PITX2 (PITX2.Q2), PAX6 (PAX6.01), SIX3 (SIX3.01) for the factors implicated in eye development [38], as well as in Hi-C conformation [41, 42]. Notably, eQTL causative SNP rs10874833, being located within the GFI1 TFBS in Alu, may disrupt the binding site of GFI1, which can be the cause of negative feedback on GFI1 expression rate reported in GTEx survey. 
Using GTEX v8 RNA-Seq profiles we also ascertained that GFI1 highest expression rate across 53 tissues available is maintained in EBV-transformed lymphocytes (cell culture): its average expression rate is $2.16 \mathrm{Tpm}$ while standard deviation being 0.64 (100 samples considered). Notably, GFI1 gene maintains high expression correlation rate with another oncogene Malat1 ncRNA, while both maintain negative correlation to TP53 (Table 3) as reported earlier [43]. It most probably reflects EBV induced immortality in cells. GFI1 expression rate in pituitary tissue is about 0.13 $( \pm 0.08) \mathrm{Tpm}$. While this value is more than a median across 53 tissues, still it's rather low for the robust assessments of its interactions/pathways, implying a necessity of addressing embryonic cells for the final verdict.

It's worth noting that pituitary gland contains stem/ progenitor cells pool [44], and anatomically is located within close vicinity of the optical chiasm [45], which may imply common mechanisms of their early embryonic development, including retinal tissues. Pituitary tissue is the top one in Malat1 expression rate throughout the 53 tissues maintained by GTEX Consortium v.8 dataset (personal observation), underlining its high stem cell specific genes turnover [43].

Still, it is necessary to state that the target enhancer score in current GeneHancer database version is small (score = 232 from 700 maximal) accounting for the distance to the target gene $(840 \mathrm{~kb})$, GFI1 small expression rate in the tissues considered (see above), sparse tissue manifestation instances and single (eQTL) source of evidence.

Along with gaining the insight into the target and mechanisms for GWAS intergenic locus supported by GTEx eQTL data considering optic disc size trait, we also performed haplotyping of GWAS encompassed locus for 4 supergroups. It was elucidated that EAS supergroup maintains the unimodal allele frequency distribution spanning 15 SNPs belonging to the major low-risk allele. But it also implies ' $G$ ' eQTL allele at causative SNP, and thus probably maximizing the optic disc size trait leading to the elevated myopia incidence observed in EAS populations [24]. Note, that on the contrary, AFR, SAS supergroups maintain elevated frequency of risk associated allele (Table 2; Fig. 3). Also, AFR supergroup maintains African- specific low frequency haplotypes with GWAS SNPs triplets AA(G/A) and causal eQTL

Table 3 Pairwise Pearson correlation rate of three genes based on 100 random samples of EBV-transformed lymphocytes RNASeq data (GTEx Consortium; v.8; https://www.gtexportal.org)

\begin{tabular}{lllll}
\hline Variables & GFI1 & TP53RK & TP53 & MALAT1 \\
\hline GFI1 & $\mathbf{1}$ & -0.222 & -0.058 & $\mathbf{0 . 3 8 6}$ \\
TP53RK & -0.222 & $\mathbf{1}$ & $\mathbf{0 . 4 1 0}$ & $\mathbf{- 0 . 4 0 5}$ \\
TP53 & -0.058 & $\mathbf{0 . 4 1 0}$ & $\mathbf{1}$ & $\mathbf{- 0 . 3 3 1}$ \\
MALAT1 & $\mathbf{0 . 3 8 6}$ & $\mathbf{- 0 . 4 0 5}$ & $\mathbf{- 0 . 3 3 1}$ & $\mathbf{1}$ \\
\hline
\end{tabular}

Values in bold are different from 0 with a significance level alpha $=0.05$
SNPs to be a 'C' variant (Table 2, Caption), which possibly impacts the elevated rate of glaucoma incidence in this group as well.

\section{Conclusion}

Besides outlining that Alu TEs are apparently involved in regulation of the target site, we report unequal frequency profiles of GWAS/eQTL enhancer-related haplotypes in continental supergroups (Table 2, Fig. 3), implying the optical disc area trait may pertain to the specific correspondent enhancer site involvement. We underscore the presence of unique European specific minor haplotype along with high homogeneity for major haplotype in East Asian population. Based on the observation, we speculate that there may be 2 alternative factors modulating enhancer effects for the eye disc area. To assess it, it would be reasonable to experimentally elaborate on haplotypes consisted of 2 tag SNPs (Table 2). It may shed the light if there are specific risks of each haplotype state.

Concerning the possible implications in other GWAS/ eQTL functional annotations, we'd underline the essential obstacles of inherently developmentally onset traits analysis rendering assessment of gene expression rate dynamics at specific tissues/organs developmental stages. Extensive chromatin remodeling dynamics during embryogenesis also adds to the complexity of developmentally onset traits analysis.

\section{Methods \\ eQTL data}

GTEx data of eQTL score profiles [17] in the region were downloaded from UCSC resource (www.genome. ucsc.edu; genome.ucsc.edu > exeqtlcluster)

GeneHancer data on the promoter and enhancer regions were downloaded from ucsc resource (www.genome.ucsc. edu; genehancer_reg_elements_doubleelite track)

\section{Genomes data}

We downloaded a subset of 1000 Genomes Consortium (1000GP) phase 3 data [46] (http://www.internationalgenome.org/) for 4 supergroups: 1) African (AFR; 504 individuals total); 2) East Asian (EAS; 504 individuals total), 3) European (EUR; 503 individuals total), 4) South Asian (SAS; 489 individuals total). We omitted American native supergroup (AMR) from 1000G since it significantly overlaps with other 4 supergroups by alleles profiles ([46], personal observation). A total of 2000 individuals were analyzed. The data are presented in Additional File 2.

\section{Retrieval and statistical tools}

We employed the PLINK toolset [47] for extracting and managing the haplotype data. We used XLStat software for Principal Component Analysis (PCA; www.xlstat. com). 


\section{Supplementary information}

Supplementary information accompanies this paper at https://doi.org/10. 1186/s12863-020-00873-z.

Additional file 1. Segment of AluJo is the preferred GFI1 binding site. Supplementary information.

Additional file 2. Data on 1000 G 15-fold SNPs haplotypes of 2000 individuals studied.

\section{Abbreviations}

TE: Transposable Elements; TFBS: Transcription Factor Binding Sites; GF/1: Growth Factor Independent 1

\section{Acknowledgments}

The authors thank anonymous referees for helpful suggestions.

\section{About this supplement}

This article has been published as part of BMC Genetics Volume 21 Supplement 1, 2020: Selected Topics in "Systems Biology and Bioinformatics" - 2019: genetics. The full contents of the supplement are available online at https://bmcgenet.biomedcentral.com/articles/supplements/volume-21supplement-1.

\section{Authors' contributions}

VNB conceived and designed the work; VNB and ROB performed the experiments; VNB, ROB and YLO analyzed the data; VNB and YLO wrote the manuscript. All authors read and approved the final manuscript.

\section{Funding}

This population genetics studies (RB) were funded by the Russian Science Foundation, grant 19-15-00219. Bioinformatics and manuscript preparation (VB, YO) were supported by Novosibirsk State University (Russian Ministry of Education). The publication cost was covered by Russian Ministry of Education and Science Project No.28.12487.2018/12.1.

The funding bodies played no role in the design of the study and collection, analysis, and interpretation of data and in writing of the manuscript.

\section{Availability of data and materials}

All data generated or analyzed in this study are publicly available: 1000 Genomes Consortium at ftp://ftp-trace.ncbi.nih.gov/1000genomes/ftp; and UCSC resource at: http://hgdownload.soe.ucsc.edu/goldenPath/hg19/ database/. Additionally, the pre-processed data for 15 SNPs and 2000 individuals from 1000GP is located in Supplementary File 2.xls.

\section{Ethics approval and consent to participate}

Not applicable.

\section{Consent for publication}

Not applicable.

\section{Competing interests}

The authors declare that they have no competing interests.

\section{Author details}

${ }^{1}$ Institute of Cytology and Genetics, Lavrentyeva 10, Novosibirsk 630090 Russia. ${ }^{2}$ Novosibirsk State University, Pirogova Str 2, Novosibirsk 630090 , Russia. ${ }^{3}$ I.M. Sechenov First Moscow State Medical University of the Ministry of Health of the Russian Federation (Sechenov University), Trubetskaya 8-2, Moscow 119991, Russia.

\section{Published: 22 October 2020}

\section{References}

1. Hindorff LA, Sethupathy P, Junkins HA, Ramos EM, Mehta JP, Collins FS, Manolio TA. Potential etiologic and functional implications of genome-wide association loci for human diseases and traits. PNAS. 2009;106(23):9362-7.

2. Shastry BS. SNPs: impact on gene function and phenotype. Methods Mol Biol. 2009;578:3-22. https://doi.org/10.1007/978-1-60327-411-1_1 Review.
3. Ernst J, Kellis M. Discovery and characterization of chromatin states for systematic annotation of the human genome. Nat Biotechnol. 2010;28(8): 817-25. https://doi.org/10.1038/nbt.1662.

4. Ernst J, Kheradpour P, Mikkelsen TS, Shoresh N, Ward LD, Epstein CB, Zhang X, Wang L, Issner R, Coyne M, Ku M, Durham T, Kellis M, Bernstein BE. Mapping and analysis of chromatin state dynamics in nine human cell types. Nature. 2011;473(7345):43-9.

5. ENCODE Project Consortium, Dunham I, Kundaje A, Aldred SF, Collins PJ, Davis CA, et al. An integrated encyclopedia of DNA elements in the human genome. Nature. 2012;489(7414):57-74.

6. Lieberman-Aiden E, van Berkum NL, Williams L, Imakaev M, Ragoczy T, Telling A, Amit I, Lajoie BR, Sabo PJ, Dorschner MO, Sandstrom R, Bernstein B, Bender MA, Groudine M, Gnirke A, Stamatoyannopoulos J, Mirny LA, Lander ES, Dekker J. Comprehensive mapping of long-range interactions reveals folding principles of the human genome. Science. 2009;326(5950): 289-93. https://doi.org/10.1126/science.1181369.

7. Thibodeau A, Uyar A, Khetan S, Stitzel ML, Ucar D. A neural network based model effectively predicts enhancers from clinical ATAC-seq samples. Sci Rep. 2018:8(1):16048. https://doi.org/10.1038/s41598-018-34420-9.

8. Melnikov A, Murugan A, Zhang X, Tesileanu T, Wang L, et al. Systematic dissection and optimization of inducible enhancers in human cells using a massively parallel reporter assay. Nat Biotechnol. 2012;30(3):271-7. https:// doi.org/10.1038/nbt.2137.

9. Visel A, Minovitsky S, Dubchak I, Pennacchio LA. VISTA enhancer browser-a database of tissue-specific human enhancers. Nucleic Acids Res. 2007; 35(Database issue):D88-92

10. Coppola CJ, Ramaker R, Mendenhall EM. Identification and function of enhancers in the human genome. Hum Mol Genet. 2016;25(R2):R190-7.

11. Fishilevich S, Nudel R, Rappaport N, Hadar R, Plaschkes I, Iny Stein T, Rosen N, Kohn A, Twik M, Safran M, Lancet D, Cohen D. GeneHancer: genomewide integration of enhancers and target genes in GeneCards. Database (Oxford). 2017;2017. https://doi.org/10.1093/database/bax028.

12. Andersson R, Gebhard C, Miguel-Escalada I, Hoof I, Bornholdt J, Boyd M, et al. An atlas of active enhancers across human cell types and tissues. Nature. 2014;507(7493):455-61. https://doi.org/10.1038/nature12787.

13. Zerbino DR, Wilder SP, Johnson N, Juettemann T, Flicek PR. The ensemble regulatory build. Genome Biol. 2015;16:56. https://doi.org/10.1186/s13059015-0621-5.

14. Khan A, Zhang X. dbSUPER: a database of super-enhancers in mouse and human genome. Nucleic Acids Res. 2016;44(D1):D164-71. https://doi.org/10. 1093/nar/gkv1002

15. Dreos R, Ambrosini G, Périer RC, Bucher P. The eukaryotic promoter database: expansion of EPDnew and new promoter analysis tools. Nucleic Acids Res. 2015;43(Database issue):D92-6. https:/doi.org/10.1093/nar/ gku1111.

16. Dimitrieva S, Bucher P. UCNEbase--a database of ultraconserved non-coding elements and genomic regulatory blocks. Nucleic Acids Res. 2013; 41(Database issue):D101-9. https://doi.org/10.1093/nar/gks1092.

17. GTEx Consortium. Human genomics. The genotype-tissue expression (GTEx) pilot analysis: multitissue gene regulation in humans. Science. 2015; 348(6235):648-60. https://doi.org/10.1126/science.1262110.

18. Kumasaka N, Knights AJ, Gaffney DJ. High-resolution genetic mapping of putative causal interactions between regions of open chromatin. Nat Genet. 2019;51(1):128-37. https://doi.org/10.1038/s41588-018-0278-6.

19. Osborne CS, Mifsud B. Capturing genomic relationships that matter Chromosom Res. 2017;25(1):15-24. https://doi.org/10.1007/s10577-016-9546-4.

20. Smemo S, Tena JJ, Kim KH, Gamazon ER, Sakabe NJ, Gómez-Marín C, et al. Obesity-associated variants within FTO form long-range functional connections with IRX3. Nature. 2014;507(7492):371-5. https://doi.org/10. 1038/nature13138.

21. Ramdas WD, van Koolwijk LM, Ikram MK, Jansonius NM, de Jong PT, Bergen AA, Isaacs A, Amin N, Aulchenko YS, Wolfs RC, Hofman A, Rivadeneira F, Oostra BA, Uitterlinden AG, Hysi P, Hammond CJ, Lemij HG, Vingerling JR, Klaver CC, van Duijn CM. A genome-wide association study of optic disc parameters. PLoS Genet. 2010;6(6):e1000978. https://doi.org/10.1371/journal. pgen.1000978.

22. Khor CC, Ramdas WD, Vithana EN, Cornes BK, Sim X, Tay WT, Saw SM, Zheng Y, Lavanya R, Wu R, Wang JJ, Mitchell P, Uitterlinden AG, Rivadeneira F, Teo YY, Chia KS, Seielstad M, Hibberd M, Vingerling JR, Klaver CC, Jansonius NM, Tai ES, Wong TY, van Duijn CM, Aung T. Genome-wide association studies in Asians confirm the involvement of ATOH7 and 
TGFBR3, and further identify CARD10 as a novel locus influencing optic disc area. Hum Mol Genet. 2011;20(9):1864-72. https://doi.org/10.1093/hmg/ ddr060.

23. Springelkamp H, Mishra A, Hysi PG, Gharahkhani P, Höhn R, Khor CC, et al. Meta-analysis of genome-wide association studies identifies novel loci associated with optic disc morphology. Genet Epidemiol. 2015;39(3):207-16. https://doi.org/10.1002/gepi.21886.

24. Springelkamp H, Iglesias Al, Mishra A, Höhn R, Wojciechowski R, Khawaja AP, et al. New insights into the genetics of primary open-angle glaucoma based on meta-analyses of intraocular pressure and optic disc characteristics. Hum Mol Genet. 2017;26(2):438-53. https://doi.org/10.1093/ hmg/ddw399.

25. Ramdas WD, van Koolwijk LM, Cree AJ, Janssens AC, Amin N, de Jong PT, Wolfs RC, Gibson J, Kirwan JF, Hofman A, Rivadeneira F, Oostra BA, Uitterlinden AG, Ennis S, Lotery AJ, Lemij HG, Klaver CC, Vingerling JR, Jansonius NM, van Duijn CM. Clinical implications of old and new genes for open-angle glaucoma. Ophthalmology. 2011;118(12):2389-97. https://doi. org/10.1016/j.ophtha.2011.05.040.

26. Jadhav U, Cavazza A, Banerjee KK, Xie H, O'Neill NK, Saenz-Vash V, Herbert Z, Madha S, Orkin SH, Zhai H, Shivdasani RA. Extensive recovery of embryonic enhancer and gene memory stored in hypomethylated enhancer DNA. Mol Cell. 2019;74(3):542-554.e5. https://doi.org/10.1016/j.molcel.2019.02.024.

27. Gilks CB, Bear SE, Grimes HL, Tsichlis PN. Progression of interleukin-2 (IL-2)dependent rat T cell lymphoma lines to IL-2-independent growth following activation of a gene (Gfi-1) encoding a novel zinc finger protein. Mol Cell Biol. 1993;13(3):1759-68.

28. Gilks CB, Porter SD, Barker C, Tsichlis PN, Gout PW. Prolactin (PRL)dependent expression of a zinc finger protein-encoding gene, Gfi-1, in Nb2 lymphoma cells: constitutive expression in autonomous sublines. Endocrinology. 1995;136(4):1805-8.

29. Wallis $D$, Hamblen M, Zhou Y, Venken $\mathrm{K}$, Schumacher A, Grimes HL, Zoghbi HY, Orkin SH, Bellen HJ. The zinc finger transcription factor Gfi1, implicated in lymphomagenesis, is required for inner ear hair cell differentiation and survival. Development. 2003;130(1):221-32.

30. Sapkota D, Chintala H, Wu F, Fliesler SJ, Hu Z, Mu X. Onecut1 and Onecut2 redundantly regulate early retinal cell fates during development. Proc Natl Acad Sci U S A. 2014;111(39):E4086-95. https:/doi.org/10.1073/pnas.1405354111.

31. Wilson NK, Timms RT, Kinston SJ, Cheng YH, Oram SH, Landry JR, Mullender J, Ottersbach K, Gottgens B. Gfi1 expression is controlled by five distinct regulatory regions spread over 100 kilobases, with Scl/Tal1, Gata2, PU.1, Erg, Meis1, and Runx1 acting as upstream regulators in early hematopoietic cells. Mol Cell Biol. 2010;30(15):3853-63. https://doi.org/10.1128/MCB.00032-10.

32. Bertolino E, Reinitz J, Manu. The analysis of novel distal Cebpa enhancers and silencers using a transcriptional model reveals the complex regulatory logic of hematopoietic lineage specification. Dev Biol. 2016:413(1):128-44. https://doi.org/10.1016/j.ydbio.2016.02.030

33. Ng FS, Calero-Nieto FJ, Göttgens B. Shared transcription factors contribute to distinct cell fates. Transcription. 2014;5(5):e978173. https://doi.org/10. 4161/21541264.2014.978173.

34. Erwin GD, Oksenberg N, Truty RM, Kostka D, Murphy KK, Ahituv N, Pollard KS, Capra JA. Integrating diverse datasets improves developmental enhancer prediction. PLoS Comput Biol. 2014;10(6):e1003677. https://doi. org/10.1371/journal.pcbi.1003677.

35. Ge SX. Exploratory bioinformatics investigation reveals importance of "junk" DNA in early embryo development. BMC Genomics. 2017;18(1):200. https:// doi.org/10.1186/s12864-017-3566-0.

36. Zhang J, Simonti CN, Capra JA. Genome-wide maps of distal gene regulatory enhancers active in the human placenta. PLoS One. 2018;13(12): e0209611. https://doi.org/10.1371/journal.pone.0209611 eCollection 2018.

37. Polak P, Domany E. Alu elements contain many binding sites for transcription factors and may play a role in regulation of developmental processes. BMC Genomics. 2006;7:133

38. Kulakovskiy IV, Vorontsov IE, Yevshin IS, Sharipov RN, Fedorova AD, Rumynskiy El, Medvedeva YA, Magana-Mora A, Bajic VB, Papatsenko DA, Kolpakov FA, Makeev VJ. HOCOMOCO: towards a complete collection of transcription factor binding models for human and mouse via large-scale ChIP-Seq analysis. Nucleic Acids Res. 2018;46(D1):D252-9. https://doi.org/10. 1093/nar/gkx1106

39. Santolini M, Mora T, Hakim V. A general pairwise interaction model provides an accurate description of in vivo transcription factor binding sites. PLoS One. 2014;9(6):e99015. https://doi.org/10.1371/journal.pone.0099015.
40. Lis $\mathrm{M}$, Walther $\mathrm{D}$. The orientation of transcription factor binding site motifs in gene promoter regions: does it matter? BMC Genomics. 2016;17:185. https://doi.org/10.1186/s12864-016-2549-x.

41. Cournac A, Koszul R, Mozziconacci J. The 3D folding of metazoan genomes correlates with the association of similar repetitive elements. Nucleic Acids Res. 2016;44(1):245-55. https://doi.org/10.1093/nar/gkv1292.

42. Gu Z, Jin K, Crabbe MJC, Zhang Y, Liu X, Huang Y, Hua M, Nan P, Zhang Z, Zhong Y. Enrichment analysis of Alu elements with different spatial chromatin proximity in the human genome. Protein Cell. 2016;4:250-66. https://doi.org/10.1007/s13238-015-0240-7.

43. Ma XY, Wang JH, Wang JL, Ma CX, Wang XC, Liu FS. Malat1 as an evolutionarily conserved IncRNA, plays a positive role in regulating proliferation and maintaining undifferentiated status of early-stage hematopoietic cells. BMC Genomics. 2015;16:676. https://doi.org/10.1186/ s12864-015-1881-X.

44. Vankelecom H, Gremeaux L. Stem cells in the pituitary gland: a burgeoning field. Gen Comp Endocrinol. 2010;166(3):478-88. https://doi.org/10.1016/j. ygcen.2009.11.007.

45. Camper SA. Beta-catenin stimulates pituitary stem cells to form aggressive tumors. Proc Natl Acad Sci U S A. 2011;108(28):11303-4. https://doi.org/10. 1073/pnas.1108275108.

46. 1000 Genomes Project Consortium, Abecasis GR, Auton A, Brooks LD, DePristo MA, Durbin RM, Handsaker RE, Kang HM, Marth GT, McVean GA. An integrated map of genetic variation from 1,092 human genomes. Nature. 2012;491 (7422):56-65.

47. Chang CC, Chow CC, Tellier LC, Vattikuti S, Purcell SM, Lee JJ. Secondgeneration PLINK: rising to the challenge of larger and richer datasets. Gigascience. 2015;4:7. https://doi.org/10.1186/s13742-015-0047-8.

\section{Publisher's Note}

Springer Nature remains neutral with regard to jurisdictional claims in published maps and institutional affiliations.

Ready to submit your research? Choose BMC and benefit from:

- fast, convenient online submission

- thorough peer review by experienced researchers in your field

- rapid publication on acceptance

- support for research data, including large and complex data types

- gold Open Access which fosters wider collaboration and increased citations

- maximum visibility for your research: over $100 \mathrm{M}$ website views per year

At $\mathrm{BMC}$, research is always in progress.

Learn more biomedcentral.com/submissions 